\title{
VARIOUS STRUCTURES IN 8-DIMENSIONAL VECTOR BUNDLES OVER 8-MANIFOLDS
}

\author{
MARTIN ČADEK \\ Department of Algebra and Geometry, Masaryk University \\ Janáčkovo nám. 2a, 66295 Brno, Czech Republic \\ E-mail: cadek@math.muni.cz \\ JIŘÍ VANŽURA \\ Academy of Sciences of the Czech Republic, Institute of Mathematics \\ Žižkova 22, 616 62 Brno, Czech Republic \\ E-mail:vanzura@ipm.cz
}

\begin{abstract}
The paper is an overview of our results concerning the existence of various structures, especially complex and quaternionic, in 8-dimensional vector bundles over closed connected smooth 8-manifolds.

1. Introduction. Let $\xi$ be an oriented $n$-dimensional vector bundle over a closed connected smooth manifold $M$ of dimension $n$. It is well known that $\xi$ is associated with a principal fiber bundle with the structure group $S O(n)$. Let $G$ be a subgroup of $S O(n)$. Under a $G$-structure on $\xi$ we understand a reduction of this principal fiber bundle to the subgroup $G$. There are plenty of interesting reductions. The most famous is a reduction to $S O(n-k)$ which means geometrically the existence of $k$ linearly independent sections in the vector bundle $\xi$. Similarly a reduction to $S O(k) \times S O(n-k)$ represents the existence of a $k$-dimensional subbundle in $\xi$. Both cases for $k \leq 3$ has been treated by many authors (see for instance $[\mathrm{AD}],[\mathrm{CS}],[\mathrm{Du}],[\mathrm{K} 1],[\mathrm{K} 2],[\mathrm{T} 2]$ ).

In this overview we are interested especially in $U(4)$-structures, which we shall call complex structures, in $S p(2)$-structures and $S p(2) \cdot S p(1)$-structures in dimension $n=8$.
\end{abstract}

1991 Mathematics Subject Classification: 57R22, 57R25, 55R25, 22E99.

Key words and phrases: vector bundle, reduction of the structure group, Cayley numbers, principle of triality, classifying spaces, characteristic classes, obstructions.

Research supported by the grant 201/96/0310 of the Grant Agency of the Czech Republic.

The paper is in final form and no version of it will be published elsewhere. 
These structures appear in the tangent bundles of the Kähler, the hyper-Kähler and the quaternion-Kähler manifolds of dimension 8, respectively.

First, we explain the necessary notions. $S p(k)$ denotes the group of the quaternionic linear automorphisms acting from the left on the right quaternionic $k$-dimensional vector space $\mathbb{H}^{k}$ preserving the canonical positive definite Hermitian form on it. If we identify $\mathbb{H}^{k}$ with $\mathbb{R}^{4 k}, S p(k)$ can be understood as a subgroup of $S O(4 k) . S p(k) \cdot S p(1)$ is the group $S p(k) \times S p(1) /\{(1,1),(-1,-1)\}$. Identifying again $\mathbb{H}^{k}$ with $\mathbb{R}^{4 k}$, the following left action on $\mathbb{H}^{k}$

$$
(A, \alpha) v=A v \bar{\alpha}, \quad A \in S p(k), \alpha \in S p(1), v \in \mathbb{H}^{k},
$$

where $\bar{\alpha}$ is the quaternionic conjugate to $\alpha$, induces an inclusion $S p(k) \cdot S p(1) \hookrightarrow S O(4 k)$.

The $S p(k) \cdot S p(1)$-structure is sometimes called a quaternionic structure since it is just the structure which appears in the tangent bundles of the quaternionic projective spaces.

Notice that the existence of reductions to the subgroups $S p(k)$ and $S p(k) \cdot S p(1)$ for $k=1$ can be easily solved since $S p(1)$ is isomorphic to $S U(2)$ and $S p(1) \cdot S p(1)$ is isomorphic to $\operatorname{Spin}(4)$.

In this paper we shall study the case $n=8$, i.e. we shall consider an oriented 8dimensional vector bundle $\xi$ over a closed connected smooth manifold $M$ of dimension 8 . The reason for this restriction is twofold. First, in dimension 8 we have at our disposal a complete classification of 8-dimensional oriented vector bundles over 8-dimensional CWcomplexes in terms of the characteristic classes. (A classification of this type is possible also in other low dimensions. For further information see [CV1].) Secondly, the group $\operatorname{Spin}(8)$ is the only group among the groups $\operatorname{Spin}(n), n=2,3, \ldots$ which admits outer automorphisms. This enables us a convenient transformation of our original problems. The problem of the reduction to a subgroup $G=U(k), S p(k)$ or $S p(k) \cdot S p(1)$ for greater $n=2 k$ or $4 k$ is in general topologically difficult. The reason, roughly speaking, is that the corresponding homogeneous spaces $S O(n) / G$ have nontrivial homotopy groups from the dimension $n / 2$.

Let us remark here that the problem of existence of a complex structure in the tangent bundle of a 4-dimensional manifold has been solved by Hirzebruch and Hopf $[\mathrm{HH}]$ and in a 6-dimensional vector bundle by C. Ehresmann [E]. Also the existence of a complex structure in 8-dimensional vector bundles over 8-manifolds has been treated. T. Heaps has established necessary and sufficient conditions for the existence of such a structure in the tangent bundles in [He]. E. Thomas in [T1] has solved the general problem of the existence of a complex structure in an even dimensional oriented vector bundle $\xi$ over an oriented 8-dimensional manifold $M$ constructing the Postnikov tower and using the method of the generating class for computing obstructions. (See [T2].) His main result contains a secondary cohomology operation applied on a class which is not easy to find. He has succeeded in computing the secondary operation only in the special case when the oriented closed manifold $M$ satisfies $\delta w_{2}(M)=0$ and the bundle satisfies $\delta w_{2}(\xi)=0$ and $w_{4}(\xi)=$ $w_{4}(M)$. In our paper we assume that the oriented closed 8-dimensional manifold $M$ satisfies $w_{2}(M)=0$ and that $w_{2}(\xi)=0$. Our necessary and sufficient conditions in this case are only in terms of the cohomology ring of $M$ without any secondary cohomology operation. 
Also our method of proof is different. We will show that in the dimension 8 the existence of a $U(4)$-structure, an $S p(2)$-structure and an $S p(2) \cdot S p(1)$-structure can be reduced to the problems of existence of a 2-dimensional subbundle, 3 linearly independent sections and a 3-dimensional subbundle in a certain other 8-dimensional vector bundle, respectively.

To prove the reduction theorem we exploit the Cayley numbers, the principle of triality and the triality automorphism of $\operatorname{Spin}(8)$. We use the triality to describe the isomorphisms between the double covering of $U(4)$ and $\operatorname{Spin}(6) \cdot \operatorname{Spin}(2)$, between $\operatorname{Sp}(2)$ and $\operatorname{Spin}(5)$, and between $\operatorname{Sp}(2) \cdot S p(1)$ and $\operatorname{Spin}(5) \cdot \operatorname{Spin}(3)$. All this is carried out in Section 2 .

In Section 3 we present results about the existence of linearly independent sections (or in other words about the existence of a $k$-field) and various subbundles in an 8-dimensional oriented vector bundle $\xi$. For the existence of a 2-dimensional subbundle and 3 linearly indpendent sections we have applied the results of M. C. Crabb and B. Steer [CS] derived using index theory. The most difficult problem was the existence of a 3-dimensional subbundle. Here it was necessary to describe the characteristic classes of 3-dimensional vector bundles over an 8-dimensional manifold. We have succeeded only under the assumption that the 3 -dimensional vector bundle is a spin bundle. Then we were able to prove a theorem about the existence of a 3-dimensional subbundle of an 8-dimensional vector bundle. Explicit computations of secondary and tertiary cohomology operations play very important role here. They are based on the characterization of oriented 8dimensional vector bundles over 8-dimensional CW-complexes and on the comparison of two different approaches to the question of the existence of 3 -fields.

The previous results are applied to obtain necessary and sufficient conditions for the existence of a $U(4)$-structure and an $S p(2)$-structure in oriented 8-dimensional spin vector bundles in Section 4. In the last section we will establish nontrivial sufficient conditions for 8-dimensional vector bundles to have an $S p(2) \cdot S p(1)$ - structure. In both sections the results are demonstrated on examples.

2. The action of $U(4), S p(2)$ and $S p(2) \cdot S p(1)$ on the Cayley numbers. The letters $\mathbb{Z}, \mathbb{R}, \mathbb{C}, \mathbb{H}$ and $\mathbb{O}$ will denote the integers, the real numbers, the complex numbers, the quaternions and the Cayley numbers, respectively.

Let $\pi: \operatorname{Spin}(8) \rightarrow S O(8)$ be the standard double covering. Since $S p(2)$ is simply connected, there is a monomorphism $\operatorname{Sp}(2) \hookrightarrow \operatorname{Spin}(8)$ covering the standard inclusion $S p(2) \hookrightarrow S O(8):$

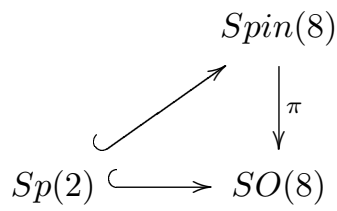

The action of $S p(2) \times S p(1)$ described in Section 1 determines a homomorphism $S p(2) \times S p(1) \rightarrow S O(8)$ with kernel $\{(1,1),(-1,-1)\}$, whence an inclusion $S p(2) \cdot S p(1) \hookrightarrow$ $S O(8)$. The former homomorphism induces also a homomorphism $S p(2) \times S p(1) \rightarrow$ 
$\operatorname{Spin}(8)$. The kernel of this homomorphism is again $\{(1,1),(-1,-1)\}$. That is why there is an inclusion $S p(2) \cdot S p(1) \hookrightarrow \operatorname{Spin}(8)$ such that the diagram

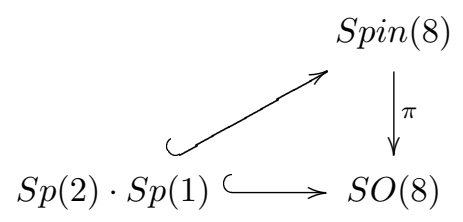

commutes.

We denote by $\tilde{U}(4)=\pi^{-1} U(4)$ the inverse image of the subgroup $U(4) \subset S O(8)$. Because the Lie group $U(4)$ is connected we can see that the Lie group $\tilde{U}(4)$ has either one or two components. It is easy to find a curve lying in $\tilde{U}(4)$ and joining the elements 1 and -1 . This shows that the group $\tilde{U}(4)$ is connected and we get the commutative diagram

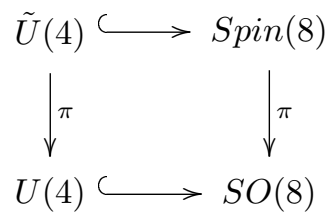

Now we convert the Cayley numbers into a right quaternionic vector space. Although $\mathbb{H}$ is a subalgebra of $\mathbb{O}$, the usual multiplication is not a right action. We define a new multiplication denoted by the $\operatorname{dot} \cdot: \mathbb{O} \times \mathbb{H} \rightarrow \mathbb{O}$ in the following way

$$
x \cdot 1=x, \quad x \cdot i=x i, \quad x \cdot j=x j, \quad x \cdot k=(x i) j,
$$

where $x \in \mathbb{O}$ and $x y$ stands for the usual multiplication in $\mathbb{O}$. This multiplication converts $\mathbb{O}$ into a right $\mathbb{H}$-vector space with the basis 1 and $e$. (The basis of $\mathbb{O}$ over $\mathbb{R}$ is $1, i, j, k, e, f, g, h$, the usual multiplication is given in the same way as in [Po].) Since the old and the new multiplication by $i$ and $j$ from the right are the same, we will omit the dot - when multiplying by these elements from the right.

This multiplication enables us to describe the Lie algebras corresponding to the groups $\tilde{U}(4), \operatorname{Sp}(1), \operatorname{Sp}(2), \operatorname{Spin}(2), \operatorname{Spin}(3), \operatorname{Spin}(5)$ and $\operatorname{Spin}(6)$ in the following way:

$$
\begin{aligned}
\mathfrak{u}(4) & =\{a \in \mathfrak{s o}(8): a(x i)=a(x) i \text { for every } x \in \mathbb{O}\}, \\
\mathfrak{s} \mathfrak{p}(1) & =\{a \in \mathfrak{s o}(8): \text { there is } \alpha \in \mathbb{H}, \bar{\alpha}=-\alpha, a(x)=x \cdot \bar{\alpha}\}, \\
\mathfrak{s p}(2) & =\{a \in \mathfrak{s o}(8): a(x i)=a(x) i, a(x j)=a(x) j \text { for every } x \in \mathbb{O}\}, \\
\mathfrak{s o}(2) & =\{c \in \mathfrak{s o}(8): c(j)=c(k)=c(e)=c(f)=c(g)=c(h)=0\}, \\
\mathfrak{s o}(3) & =\{c \in \mathfrak{s o}(8): c(k)=c(e)=c(f)=c(g)=c(h)=0\}, \\
\mathfrak{s o}(5) & =\{c \in \mathfrak{s o}(8): c(1)=c(i)=c(j)=0\}, \\
\mathfrak{s o}(6) & =\{c \in \mathfrak{s o}(8): c(1)=c(i)=0\} .
\end{aligned}
$$

According to $[\mathrm{Fr}]$ (see also $[\mathrm{Br}]$ ) there are outer automorphisms $\lambda$ and $\kappa$ of $\mathfrak{s o}(8)$ such that the principle of triality holds. For every $x, y \in \mathbb{O}$ and every $a \in \mathfrak{s o}(8)$ we have

$$
a(x y)=b(x) y+x c(y)
$$


where

$$
b=(\lambda \kappa)(a), \quad c=(\kappa \lambda)(a) .
$$

The automorphisms $\lambda$ and $\kappa$ are described in detail in [Fr] and [Bra]. For the moment we need only the following properties

$$
\lambda^{3}=\mathrm{id}, \quad \kappa^{2}=\mathrm{id}, \quad \kappa \lambda \kappa=\lambda^{2}, \quad \lambda \neq \mathrm{id} .
$$

Let us define $\operatorname{Spin}(6) \cdot \operatorname{Spin}(2)=\operatorname{Spin}(6) \times \operatorname{Spin}(2) /\{(1,1),(-1,-1)\}$ and $\operatorname{Spin}(5)$. $\operatorname{Spin}(3)=\operatorname{Spin}(5) \times \operatorname{Spin}(3) /\{(1,1),(-1,-1)\}$. It is not difficult to prove (see [CV3] and [CV5]):

LEMMA 2.1. The automorphism $\kappa \lambda$ maps the Lie algebras $\mathfrak{u}(4), \mathfrak{s p}(2), \mathfrak{s p}(2) \oplus \mathfrak{s p}(1)$ on the Lie algebras $\mathfrak{s o}(2) \oplus \mathfrak{s o}(6), \mathfrak{s o}(5)$ and $\mathfrak{s o}(3) \oplus \mathfrak{s o}(5)$, respectively. Taking this automorphism on the level of the Lie group Spin(8), its appropriate restrictions give isomorphisms between $\hat{U}(4)$ and $\operatorname{Spin}(6) \cdot \operatorname{Spin}(2)$, between $\operatorname{Sp}(2)$ and $\operatorname{Spin}(5)$ and between $\operatorname{Sp}(2) \cdot \operatorname{Sp}(1)$ and $\operatorname{Spin}(5) \cdot \operatorname{Spin}(3)$.

Since the $G$-structures with $G$ equal to $\operatorname{Spin}(6) \cdot \operatorname{Spin}(2), \operatorname{Spin}(5)$ and $\operatorname{Spin}(5) \cdot \operatorname{Spin}(3)$ describe the existence of 2 -dimensional subbundles, 3 linearly independent sections and 3-dimensional subbundles in 8-dimensional spin vector bundles, respectively, we obtain immediately

TheOREM 2.2 (see [CV3] and [CV5]). Let X be a $C W$-complex and let $\xi$ be an oriented 8 -dimensional vector bundle over $X$. Then $\xi$ has an $S p(2)$-structure if and only if it has a spinor structure $\bar{\xi}$ and the vector bundle $\pi_{*}(\kappa \lambda)_{*}(\bar{\xi})$ has 3 linearly independent sections. $\xi$ has an $S p(2) \cdot S p(1)$-structure if and only if it has a spinor structure $\bar{\xi}$ and the vector bundle $\pi_{*}(\kappa \lambda)_{*}(\bar{\xi})$ has an oriented 3-dimensional subbundle.

Finally, suppose that $\xi$ has a spinor structure $\bar{\xi}$. Then $\xi$ has a $U(4)$-structure if and only if the vector bundle $\pi_{*}(\kappa \lambda)_{*}(\bar{\xi})$ has an oriented 2-dimensional subbundle.

3. Existence of sections and subbundles. Before we can formulate the existence results we must introduce the necessary notions. We will denote $w_{s}(\zeta)$ the $s$-th StiefelWhitney class of the vector bundle $\zeta, p_{s}(\zeta)$ the $s$-th Pontrjagin class, and $e(\zeta)$ the Euler class. If $M$ is a manifold, the notation $w_{s}(M), p_{s}(M)$ and $e(M)$ will stand for the characteristic classes of the tangent bundle of $M$. We will also use the Steenrod operations $S q^{i}: H^{n}\left(X ; \mathbb{Z}_{2}\right) \rightarrow H^{n+i}\left(X ; \mathbb{Z}_{2}\right)$ and $P_{3}^{i}: H^{n}\left(X ; \mathbb{Z}_{3}\right) \rightarrow H^{n+4 i}\left(X ; \mathbb{Z}_{3}\right)$.

Under "spin vector bundles" we understand vector bundles which admit a spin structure, i.e. vector bundles $\zeta$ satisfying $w_{1}(\zeta)=w_{2}(\zeta)=0$. Similarly, a smooth manifold $M$ with $w_{1}(M)=w_{2}(M)=0$ will be called a spin manifold. Our theorems will be formulated for spin vector bundles and this is the reason why (following [T3] and [Q]) we shall introduce further characteristic classes

$$
q_{1}(\zeta) \in H^{4}(M ; \mathbb{Z}), q_{2}(\zeta) \in H^{8}(M ; \mathbb{Z}), \text { and } \varepsilon(\zeta) \in H^{8}\left(M ; \mathbb{Z}_{2}\right)
$$

sometimes called spin characteristic classes of a spin vector bundle $\zeta$ over $M$. We get them often by a mere algebraic manipulation with the Pontrjagin classes (which of course would not be possible if the bundle $\zeta$ were not a spin bundle). Since the base $M$ of the vector bundle $\zeta$ is an oriented 8-dimensional closed connected manifold, the spin characteristic 
classes $q_{1}(\zeta), q_{2}(\zeta)$ and $\varepsilon(\zeta)$ can be defined uniquely (see [CV2]) and under the additional assumption that $H^{4}(M ; \mathbb{Z})$ has no element of order 4 they are uniquely determined by the equations

$$
\begin{gathered}
p_{1}(\zeta)=2 q_{1}(\zeta), \quad \rho_{2} q_{1}(\zeta)=w_{4}(\zeta), \\
p_{2}(\zeta)=q_{1}^{2}(\zeta)+2 e(\zeta)+4 q_{2}(\zeta), \quad \varepsilon(\zeta)=\rho_{2} q_{2}(\zeta),
\end{gathered}
$$

where $\rho_{2}$ denotes the reduction mod 2. (The introduction of these classes is a little more complicated if the base of the vector bundle $\zeta$ is a CW-complex. See [T3] and [CV2].) The letters $q_{1}, q_{2}, e$ and $w_{s}$ will be used for the characteristic classes of the universal 8-dimensional spin vector bundle over the classifying space $B \operatorname{Spin}(8)$. Finally, let us recall that every 3 -dimensional spin vector bundle $\eta$ over a CW-complex $X$ has the first Pontrjagin class of the form

$$
p_{1}(\eta)=4 a
$$

for some $a \in H^{4}(X ; \mathbb{Z})$. It is easily seen from the relation

$$
\rho_{4} p_{1}(\zeta)=\mathfrak{P} w_{2}(\zeta)+i_{*} w_{4}(\zeta)
$$

where $\rho_{4}$ is reduction $\bmod 4, \mathfrak{P}: H^{2 k}\left(X ; \mathbb{Z}_{2}\right) \rightarrow H^{4 k}\left(X ; \mathbb{Z}_{4}\right)$ is the Pontrjagin square and $i: \mathbb{Z}_{2} \rightarrow \mathbb{Z}_{4}$ is a monomorphism.

First, we state the theorem on the existence of 2-dimensional subbundles which is the application of Theorem 0.4 from [CS] to dimension 8. See also [CV2].

THEOREM 3.1. Let $M$ be a closed connected smooth spin manifold of dimension 8 and let $\zeta$ be an 8-dimensional spin vector bundle over $M$. Then in $\zeta$ there exists a 2dimensional subbundle whose Euler class is $u$ if and only if there is $v \in H^{6}(M ; \mathbb{Z})$ such that

(i) $\rho_{2} v=w_{6}(\zeta)+w_{4}(\zeta) \rho_{2} u+\rho_{2} u^{3}$ and $u v=e(\zeta)$,

(ii) $\left\{q_{1}(M) q_{1}(\zeta)-q_{1}^{2}(\zeta)-2 q_{2}(\zeta)\right\}[M] \equiv 0 \bmod 4$.

Remark 3.2. In terms of the Pontrjagin classes and the Euler class, the latter condition can be expressed

$$
\left\{2 p_{1}(M) p_{1}(\zeta)-p_{1}^{2}(\zeta)-4 p_{2}(\zeta)+8 e(\zeta)\right\}[M] \equiv 0 \bmod 32 .
$$

In order to establish the existence of an $S p(2)$-structure, we shall need a result on the existence of 3 linearly independent sections. Applying Theorem 0.4 from [CS] to the dimension 8 we get

Theorem 3.3 ([CV3], Corollary 5.3). Let $\zeta$ be an 8-dimensional spin vector bundle over a closed connected smooth spin manifold $M$ of the same dimension. Then $\zeta$ has three linearly independent sections if and only if

(1) $w_{6}(\zeta)=0$,

(2) $e(\zeta)=0$

(3) $\left\{q_{1}(M) q_{1}(\zeta)-q_{1}^{2}(\zeta)-2 q_{2}(\zeta)\right\}[M] \equiv 0 \bmod 8$.

Further applications of $[\mathrm{CS}]$ and $[\mathrm{Du}]$ provide necessary and sufficient conditions for a given 3-dimensional spin vector bundle $\eta$ to be a subbundle of a given 8-dimensional spin vector bundle $\zeta$ over a closed connected spin manifold $M$. 
To solve the problem of the existence of $S p(2) \cdot S p(1)$-structures we would need to answer the question whether a given 8-dimensional spin vector bundle has a 3-dimensional (not necessarily spin) subbundle. However, we are not able to describe the characteristic classes of all 3-dimensional vector bundles over a given manifold $M$ of dimension 8 . In Theorem 3.4 we describe only the cohomology classes which can appear as characteristic classes of a 3-dimensional spin vector bundle. Consequently, we are not able to answer the question above in full generality. In Theorem 3.6 we get only necessary and sufficient conditions for a given 8-dimensional vector bundle to have a 3-dimensional spin subbundle.

Before we state these results, we shall introduce higher order cohomology operations which appear in Theorem 3.4.

Let $X$ be an 8-dimensional CW-complex. On $H^{4}(X ; \mathbb{Z})$ we have

$$
S q^{2} \circ S q^{2} \rho_{2}=0 \text {. }
$$

We denote by $\Sigma$ the secondary operation associated with this relation. $\Sigma$ is defined on the subgroup

$$
\operatorname{Def}(\Sigma, X)=\left\{x \in H^{4}(X ; \mathbb{Z}) ; S q^{2} \rho_{2} x=0\right\}
$$

and has values in the factor group of $H^{7}\left(X ; \mathbb{Z}_{2}\right)$ by the subgroup

$$
\operatorname{Indet}(\Sigma, X)=S q^{2} H^{5}\left(X ; \mathbb{Z}_{2}\right),
$$

and we again consider the values of $\Sigma$ as the corresponding cosets.

Let $\Phi$ be the tertiary cohomology operation associated with the relation

$$
i_{*} S q^{2} \circ \Sigma=0
$$

in dimension 4 , and uniquely determined by the properties

$$
\Phi(r)=0 \quad \text { and } \Phi(2 r)=-\rho_{4} r^{2}
$$

where $r \in H^{4}(B \operatorname{Spin}(3) ; \mathbb{Z})$ and $4 r$ is the first Pontrjagin class of the universal 3-dimensional spin vector bundle over $B \operatorname{Spin}(3)$. The operation $\Phi$ is defined on $\operatorname{Def}(\Phi, X)=\{x \in$ $\left.H^{4}(X ; \mathbb{Z}) ; S q^{2} \rho_{2} x=0, \Sigma(x) \ni 0\right\}$ and its indeterminacy is given by a certain secondary cohomology operation. More details can be found in [CV4].

Using the Postnikov-Moore decomposition and the operations above we obtain the following description of characteristic classes of 3-dimensional spin vector bundles over 8-complexes.

Theorem 3.4 ([CV4], Theorem 3.10). Let $X$ be an 8-dimensional $C W$-complex, and let $a \in H^{4}(X ; \mathbb{Z})$. Then there exists a 3-dimensional spin vector bundle $\eta$ over $X$ with $p_{1}(\eta)=4 a$ if and only if the following conditions are satisfied:

(i) $S q^{2} \rho_{2} a=0$,

(ii) $0 \in \Sigma(a)$,

(iii) $P_{3}^{1} \rho_{3} a+\rho_{3} a^{2}=0$,

(iv) $0 \in \Phi(a)$.

The operations $\Sigma$ and $\Phi$ can be computed on closed spin manifolds in a similar way as $\Omega$. (See [CV4], Section 5.) 
THEOREM 3.5. Let $M$ be a closed connected smooth spin manifold of dimension 8. Then

$$
\Sigma(z)=S q^{2} H^{5}\left(M ; \mathbb{Z}_{2}\right)
$$

for every $z \in \operatorname{Def}(\Sigma, M), \operatorname{Indet}(\Phi, M)=0$ and

$$
\Phi(z)=\rho_{4} \frac{1}{2}\left\{z q_{1}(M)-z^{2}\right\} .
$$

for every $z \in \operatorname{Def}(\Phi, M)$.

This assertion implies that $0 \in \Sigma(z)$.

The Postnikov-Moore decomposition and the previous results give

Theorem 3.6 ([CV4], Theorem 6.1). Let $\zeta$ be an 8-dimensional spin vector bundle over a closed connected smooth spin 8-manifold $M$ and let $R \in H^{4}(M ; \mathbb{Z})$. Then $\zeta$ has an oriented 3-dimensional spin subbundle $\eta$ with $p_{1}(\eta)=4 R$ if and only if

(i) $S q^{2} \rho_{2} R=0$,

(ii) $P_{3}^{1} \rho_{3} R+\rho_{3} R^{2}=0$,

(iii) $\left\{R q_{1}(M)-R^{2}\right\}[M] \equiv 0 \bmod 8$,

(iv) $w_{6}(\zeta)=0$,

(v) $e(\zeta)=0$,

(vi) $\left\{q_{1}^{2}(\zeta)-q_{1}(M) q_{1}(\zeta)+2 q_{2}(\zeta)+2 R^{2}+2 R q_{1}(\zeta)+2 R q_{1}(M)\right\}[M] \equiv 0 \bmod 8$.

Theorem 3.1, Theorem 3.3 and Theorem 3.6 will be used directly in the next sections when we will treat $U(4), S p(2)$ and $S p(2) \cdot S p(1)$-structures. However, the methods developed for the derivation of the results above can be applied to the problem of existence of 4 linearly independent sections and 4-dimensional subbundles in 8-dimensional spin vector bundles over 8-dimensional closed spin manifolds.

Theorem 3.7 ([CV6], Corollary 4.2). Let $\zeta$ be an 8-dimensional spin vector bundle over a closed connected smooth spin 8-manifold $M$. Then $\zeta$ has 4 linearly independent sections if and only if there is $S \in H^{4}(M ; \mathbb{Z})$ and the following conditions are satisfied:

(1) $w_{6}(\zeta)=0, S q^{2} \rho_{2} S=0$,

(2) $e(\zeta)=0$

(3) $4 p_{2}(\zeta)-p_{1}^{2}(\zeta)=16 S^{2}-8 p_{1}(\zeta) S$,

(4) $\left\{2 p_{1}(M) p_{1}(\zeta)-p_{1}^{2}(\zeta)-4 p_{2}(\zeta)\right\}[M] \equiv 0 \bmod 64$,

(5) $\left\{p_{1}(M) S-2 S^{2}\right\}[M] \equiv 0 \bmod 16$,

(6) $P_{3}^{1} \rho_{3} S+\rho_{3} S^{2}=0$.

Theorem 3.8 ([CV6], Theorem 4.4). Let $M$ be a closed connected smooth spin manifold of dimension 8 such that $H^{4}(M ; \mathbb{Z})$ has no element of order 4 . Let $\zeta$ be an 8 dimensional spin vector bundle over $M$. Then $\zeta$ is the sum of two 4-dimensional spin vector bundles if and only if there are $S_{1}, S_{2}, Q_{1}, Q_{2} \in H^{4}(M ; \mathbb{Z})$ and the following conditions are satisfied for $n=1,2$ :

(1) $p_{1}(\zeta)=2\left(Q_{1}+Q_{2}\right)$,

(2) $e(\zeta)=\left(2 S_{1}-Q_{1}\right)\left(2 S_{2}-Q_{2}\right)$,

(3) $p_{2}(\zeta)=\left(2 S_{1}-Q_{1}\right)^{2}+\left(2 S_{2}-Q_{2}\right)^{2}+4 Q_{1} Q_{2}$, 
(4) $S q^{2} \rho_{2} Q_{n}=S q^{2} \rho_{2} S_{n}=0$,

(5) $\left\{S_{n} p_{1}(M)-2 S_{n}^{2}\right\}[M] \equiv 0 \bmod 16$,

(6) $\left\{4 S_{n}^{2}-4 Q_{n} S_{n}\right\}[M] \equiv\left\{Q_{n} p_{1}(M)-2 Q_{n}^{2}\right\}[M] \bmod 16$,

(7) $P_{3}^{1} \rho_{3} Q_{n}+\rho_{3} Q_{n}^{2}=\rho_{3}\left(S_{n}^{2}-Q_{n} S_{n}\right)$,

(8) $P_{3}^{1} \rho_{3} S_{n}+\rho_{3} S_{n}^{2}=0$.

For proofs, further consequences and applications we refer the reader to the paper [CV6].

4. Existence of $U(4)$ and $S p(2)$-structures. In this section we will apply Theorem 2.2 and the results from the previous section to obtain necessary and sufficient conditions for the existence of a $U(4)$-structure and an $S p(2)$-structure in an oriented 8-dimensional spin vector bundle $\xi$ over an 8-manifold.

First we need to compute the characteristic classes of the vector bundle $\pi_{*}(\kappa \lambda)_{*} \bar{\xi}$ from the characteristic classes of $\xi$. Denote the selfmaps of the classifying space BSpin(8) induced by the automorphisms $\kappa$ and $\lambda$ of $\operatorname{Spin}(8)$ by the same letters.

Lemma 4.1. For $\kappa: B \operatorname{Spin}(8) \rightarrow B \operatorname{Spin}(8)$ and $\lambda: B \operatorname{Spin}(8) \rightarrow B \operatorname{Spin}(8)$ we have

$$
\begin{aligned}
\kappa^{*}\left(q_{1}\right) & =q_{1}, & \lambda^{*}\left(q_{1}\right) & =q_{1}, \\
\kappa^{*}\left(q_{2}\right) & =q_{2}+e, & \lambda^{*}\left(q_{2}\right) & =-e-q_{2}, \\
\kappa^{*}(e) & =-e, & \lambda^{*}(e) & =q_{2} .
\end{aligned}
$$

The proof is based on the fact that characteristic classes can be expressed as polynomials in a dual basis of the Cartan subalgebra of $\mathfrak{s o}(8)(\mathrm{see}[\mathrm{BH}])$ and on the way how $\kappa$ and $\lambda$ act on simple roots. For details see [GG] or [CV3].

THEOREM 4.2. Let $\xi$ be an 8-dimensional spin vector bundle over a closed connected smooth spin manifold $M$ of dimension 8. Then in $\xi$ there exists a complex vector bundle structure if and only if there are $u \in H^{2}(M ; \mathbb{Z})$ and $v \in H^{6}(M ; \mathbb{Z})$ such that

(i) $\rho_{2} v=w_{6}(\xi)+w_{4}(\xi) \rho_{2} u+\rho_{2} u^{3}$ and $16 u v=-4 p_{2}(\xi)+p_{1}^{2}(\xi)+8 e(\xi)$,

(ii) $\left\{p_{1}^{2}(\xi)-p_{1}(\xi) p_{1}(M)+8 e(\xi)\right\}[M] \equiv 0 \bmod 16$.

Proof. Let us choose $\bar{\xi} \in[M, B \operatorname{Spin}(8)]$ such that $\pi_{*} \bar{\xi}=\xi \in[M, B S O(8)]$, and consider the bundle $\zeta=\pi_{*}(\kappa \lambda)_{*} \bar{\xi} \in[M, B S O(8)]$. According to Theorem 2.2, the vector bundle $\xi$ has a $U(4)$-structure if and only if the vector bundle $\zeta$ has an oriented 2dimensional subbundle.

By virtue of Lemma 4.1 we have $\lambda^{*} \kappa^{*}\left(q_{1}\right)=q_{1}$, which implies $\lambda^{*} \kappa^{*}\left(w_{4}\right)=\lambda^{*} \kappa^{*} \rho_{2}\left(q_{1}\right)$ $=\rho_{2}\left(q_{1}\right)=w_{4}$. Further, $\lambda^{*} \kappa^{*}\left(w_{6}\right)=\lambda^{*} \kappa^{*} S q^{2}\left(w_{4}\right)=S q^{2}\left(w_{4}\right)=w_{6}$. Consequently, we get

$$
q_{1}(\zeta)=q_{1}(\xi), \quad w_{4}(\zeta)=w_{4}(\xi), \quad w_{6}(\zeta)=w_{6}(\xi) .
$$

Similarly, we have $\lambda^{*} \kappa^{*}\left(q_{2}\right)=-e$ and $\lambda^{*} \kappa^{*}(e)=-q_{2}$, which implies

$$
q_{2}(\zeta)=-e(\xi), \quad e(\zeta)=-q_{2}(\xi)
$$

Expressing the characteristic classes of $\zeta$ in the conditions (i) and (ii) of Theorem 3.1 in terms of the characteristic classes of $\xi$, we obtain the two conditions of the theorem. We can also see that the proof does not depend on the choice of the spin structure $\bar{\xi}$. 
In [T1] explicit necessary and sufficient conditions for the existence of a complex structure are given only for 8-dimensional vector bundles $\xi$ satisfying the conditions

$$
\delta w_{2}(\xi)=0, \quad w_{4}(\xi)=w_{4}(M) .
$$

In [CV1] we have classified oriented 8-dimensional vector bundles over CW-complexes of the same dimension (which satisfy some mild conditions on cohomology) in terms of characteristic classes. Now Theorem 4.2 enables us to decide which of these vector bundles over spin manifolds with $w_{2}=0$ have a complex structure. We will show it on the example of $G_{4,2}(\mathbb{C})$.

EXAMPLE 4.3. We shall consider the complex Grassmann manifold $G_{4,2}(\mathbb{C})$. Let us recall that $H^{*}\left(G_{4,2}(\mathbb{C}) ; \mathbb{Z}\right) \cong \mathbb{Z}\left[x_{1}, x_{2}\right] /\left(x_{1}^{3}-2 x_{1} x_{2}, x_{2}^{2}-x_{1}^{2} x_{2}\right)$. The isomorphism is given by $x_{1} \mapsto c_{1}, x_{2} \mapsto c_{2}$, where $c_{1}$ and $c_{2}$ are the Chern classes of the canonical complex vector bundle $\gamma_{2}$ over $G_{4,2}(\mathbb{C})$. A standard computation shows that

$$
\begin{gathered}
c_{1}(M)=4 c_{1}, \quad c_{2}(M)=7 c_{1}^{2}, \quad c_{3}(M)=12 c_{1} c_{2}, \quad c_{4}(M)=6 c_{1}^{2} c_{2}, \\
p_{1}(M)=2 c_{1}^{2}, \quad p_{2}(M)=14 c_{1}^{2} c_{2}, \quad e(M)=6 c_{1}^{2} c_{2},
\end{gathered}
$$

where $M=G_{4,2}(\mathbb{C})$. We can immediately see that $G_{4,2}(\mathbb{C})$ is a spin manifold.

Let $\xi$ be a spin vector bundle over $G_{4,2}(\mathbb{C})$ (i.e. $w_{2}(\xi)=0$ ). According to [CV1] $\xi$ is uniquely determined by the following characteristic classes

$$
p_{1}(\xi)=2 a c_{1}^{2}+2 b c_{2}, \quad p_{2}(\xi)=C c_{1}^{2} c_{2}, \quad e(\xi)=D c_{1}^{2} c_{2} .
$$

Further, let us write

$$
u=k c_{1} \in H^{2}\left(G_{4,2}(\mathbb{C}) ; \mathbb{Z}\right), \quad v=l c_{1} c_{2} \in H^{6}\left(G_{4,2}(\mathbb{C}) ; \mathbb{Z}\right) .
$$

Easy computation shows that the conditions (i) and (ii) of Theorem 4.2 have the form

(i) $l \equiv(k+1) b \bmod 2, \quad 4 k l=-C+2 a^{2}+2 a b+b^{2}+2 D$,

(ii) $2 a^{2}+2 a b+b^{2}-2 a-b-2 D \equiv 0 \bmod 4$.

After some simplification of (i) we can conclude that on a vector bundle $\xi$ over $G_{4,2}(\mathbb{C})$ there exists a complex vector bundle structure if and only if

$$
\begin{gathered}
8 \mid\left(-C+2 a^{2}+2 a b+b^{2}+2 D\right), \\
2 a^{2}+2 a b+b^{2}-2 a-b-2 D \equiv 0 \bmod 4 .
\end{gathered}
$$

This example also enables us to test our theorem. Taking $\xi=T\left(G_{4,2}(\mathbb{C})\right)$, the tangent bundle of $G_{4,2}(\mathbb{C})$, we can easily find that the conditions of Theorem 4.2 are satisfied. This corresponds to the fact that $G_{4,2}(\mathbb{C})$ is a complex manifold.

The main result concerning the existence of an $S p(2)$-structure is the following:

THEOREM 4.6. Let $\xi$ be an oriented 8-dimensional vector bundle over a closed connected smooth spin manifold $M$ of the same dimension. Then $\xi$ has an $S p(2)$-structure if and only if

(1) $w_{2}(\xi)=w_{6}(\xi)=0$,

(2) $\left\{4 p_{2}(\xi)-p_{1}^{2}(\xi)-8 e(\xi)\right\}[M]=0$,

(3) $\left\{p_{1}(M) p_{1}(\xi)-p_{1}^{2}(\xi)+8 e(\xi)\right\}[M] \equiv 0 \bmod 32$. 
Proof. According to Theorem 2.2 a vector bundle $\xi$ has an $S p(2)$-structure if and only if it has a spinor structure $\bar{\xi}$ and the vector bundle $\zeta=\pi_{*}(\kappa \lambda)_{*}(\bar{\xi})$ has three linearly independent sections. The characteristic classes of $\zeta$ have been computed in the proof of Theorem 4.2. So we can apply directly Theorem 3.3. Hence using the definitions of $q_{1}$ and $q_{2}$, the conditions of Theorem 3.3 for $\zeta$ read as conditions (1) - (3) of this Theorem for $\xi$.

As a consequence for the tangent bundles we get

Corollary 4.7. A closed connected smooth manifold $M$ of dimension 8 has an $S p(2)$ structure if and only if

(i) $w_{2}(M)=w_{6}(M)=0$,

(ii) $\left\{4 p_{2}(M)-p_{1}^{2}(M)-8 e(M)\right\}[M]=0$,

(iii) $e(M)[M] \equiv 0 \bmod 4$.

Since $S p(2) \hookrightarrow U(4)$ there is a natural question when a complex structure can be reduced to an $S p(2)$-structure. This is the answer.

COROLlaRY 4.8. Let $\xi$ be a complex vector bundle of complex dimension 4 over a closed connected complex spin manifold $M$ of the same dimension. Then $\xi$ has Sp(2)-structure with given underlying complex structure if and only if

(i) $c_{1}(\xi)=c_{3}(\xi)=0$,

(ii) $\left\{2 c_{2}(M) c_{2}(\xi)-2 c_{2}^{2}(\xi)-c_{1}^{2}(M) c_{2}(\xi)+4 c_{4}(\xi)\right\}[M] \equiv 0 \bmod 16$

The proof is not an immediate consequence of Theorem 4.6 and needs some extra effort. See [CV3], Corollary 5.6.

EXAMPLE 4.9. We will proceed with Example 4.3 and determine which 8-dimensional spin vector bundles over $G_{4,2}(\mathbb{C})$ have an $S p(2)$-structure. Consider again a vector bundle $\xi$ the characteristic classes of which are described in the same way as in Example 4.3. Moreover, we have

$$
w_{6}(\xi)=S q^{2} \rho_{2}\left(a c_{1}^{2}+b c_{2}\right)=\rho_{2} b c_{1} c_{2} .
$$

Hence, according to Theorem 4.6, necessary and sufficient conditions for the existence of an $S p(2)$-structure in $\xi$ are

$$
\begin{gathered}
b \equiv 0 \bmod 2 \\
C=2 a^{2}+2 a b+b^{2}+2 D \\
2 a^{2}+2 a b+b^{2}-2 a-b-2 D \equiv 0 \bmod 8 .
\end{gathered}
$$

Notice that (4.11) and (4.12) imply conditions (4.4) and (4.5) for the existence of a complex structure. Testing the tangent bundle of $G_{4,2}(\mathbb{C})$, we verify immediately that it satisfies (4.10) and (4.11) but not (4.12). So $G_{4,2}(\mathbb{C})$ does not admit an $S p(2)$-structure.

Further examples will be examined in the next section jointly with examples of $S p(2)$. $S p(1)$-structures.

5. Existence of $S p(2) \cdot S p(1)$-structure. Dealing with $S p(2) \cdot S p(1)$-structures, the starting point of our considerations is again Theorem 2.2 which converts the problem of 
the existence of an $S p(2) \cdot S p(1)$-structure in a vector bundle $\xi$ to the problem of the existence of a 3-dimensional subbundle in the vector bundle $\zeta=\pi_{*}(\kappa \lambda)_{*} \bar{\xi}$. However, the situation here is more difficult than in the cases of $U(4)$ and $S p(2)$-structures.

The difficulties consist in the fact that we are not able to answer the question whether a given 8-dimensional spin vector bundle over $M$ has a 3-dimensional oriented subbundle, we know only whether it has a 3 -dimensional spin subbundle (Theorem 3.6).

This is the reason why our conditions for manifolds with $H^{2}\left(M ; \mathbb{Z}_{2}\right) \neq 0$ are only sufficient ones.

THEOREM 5.1. Let $\xi$ be an oriented 8-dimensional vector bundle over a closed connected smooth spin manifold $M$. If there is $R \in H^{4}(M ; \mathbb{Z})$ such that the conditions

(1) $S q^{2} \rho_{2} R=0$,

(2) $\left\{R p_{1}(M)-2 R^{2}\right\}[M] \equiv 0 \bmod 16$,

(3) $w_{2}(\xi)=0$

(4) $w_{6}(\xi)=0$

(5) $4 p_{2}(\xi)-p_{1}^{2}(\xi)-8 e(\xi)=0$,

(6) $\left\{p_{1}^{2}(\xi)-p_{1}(M) p_{1}(\xi)-8 e(\xi)+8 R^{2}+4 R p_{1}(\xi)+4 R p_{1}(M)\right\}[M] \equiv 0 \bmod 32$

are satisfied, then the structure group of $\xi$ can be reduced to $S p(2) \cdot S p(1)$.

If $H^{2}\left(M ; \mathbb{Z}_{2}\right)=0$, then all the previous conditions are also necessary. The conditions (3) and (5) are necessary even if $H^{2}\left(M ; \mathbb{Z}_{2}\right) \neq 0$.

P r o of. Using Theorem 2.2 and Lemma 4.1, it is sufficient to prove that the conditions of this Theorem for a vector bundle $\xi$ imply that $\xi$ has a spinor structure $\bar{\xi}$ and that the conditions in Theorem 3.6 for the vector bundle $\zeta=\pi_{*}(\kappa \lambda)^{*} \bar{\xi}$ are satisfied for some $\bar{R}$.

The condition (i) is the same as (1). (iii) is equivalent to $(2)$. Since $q_{1}(\zeta)=q_{1}(\xi)$, (iv) is equivalent to (4). (5) means $q_{2}(\xi)=0$, which reads as (v) of Theorem 3.6. Rewriting (6) in terms of $\zeta$, we get (vi).

It remains the condition (ii) of Theorem 3.6. It need not be satisfied for a given $R$ but it is certainly satisfied for $\bar{R}=-15 R$. Moreover, if $R$ satisfies the conditions (i), (iii) and (vi) of Theorem 3.6, then $\bar{R}$ satisfies them as well since

$$
-15 \equiv(-15)^{2} \equiv 1 \bmod 16 .
$$

This completes the proof.

In the case when the tangent bundle of a manifold admits an $S p(2) \cdot S p(1)$-structure, we speak about an almost quaternionic structure. The application of Theorem 5.1 to the tangent bundles yields

COROLlaRY 5.2. Let $M$ be an oriented closed connected smooth manifold of dimension 8. If

(a) $w_{2}(M)=0$,

(b) $w_{6}(M)=0$,

(c) $4 p_{2}(M)-p_{1}^{2}(M)-8 e(M)=0$,

and there is $R \in H^{4}(M ; \mathbb{Z})$ such that

(d) $S q^{2} \rho_{2} R=0$, 
(e) $\left\{R p_{1}(M)-2 R^{2}\right\}[M] \equiv 0 \bmod 16$,

(f) $\left\{R^{2}+R p_{1}(M)-e(M)\right\}[M] \equiv 0 \bmod 4$,

then $M$ has an almost quaternionic structure. Conditions $(a)$ and $(c)$ are always necessary for the existence of this structure while the remaining ones are necessary if $H^{2}\left(M ; \mathbb{Z}_{2}\right)$ $=0$.

We can give also nontrivial sufficient conditions for the existence of an almost quaternionic structure only in terms of characteristic classes without any reference to an element $R \in H^{4}(M ; \mathbb{Z})$.

Corollary 5.3. Let $M$ be an oriented closed connected smooth manifold of dimension 8. If
(A) $w_{2}(M)=0$,
(B) $w_{6}(M)=0$,
(C) $4 p_{2}(M)-p_{1}^{2}(M)-8 e(M)=0$,
(D) $\left\{k p_{1}^{2}(M)+4 e(M)\right\}[M] \equiv 0 \bmod 16, \quad$ for $k=0$ or 1 ,

then $M$ has an almost quaternionic structure.

Proof. It is sufficient to show that the assumptions of Corollary 5.3 imply the assumptions of Corollary 5.2 for $R=0$ or $R=q_{1}(M)$.

Notice that if the assumptions of Corollary 5.3 are satisfied with $k=0$ then, according to Corollary 4.7, the manifold $M$ admits even an $S p(2)$-structure.

EXAMPLE 5.4. We return to the complex Grassmann manifold $G_{4,2}(\mathbb{C})$. It is known to be a quaternion-Kähler manifold, so it must have an almost quaternionic structure. It is easy to verify that the conditions of Corollary 5.3 are satisfied.

Further, let us consider again an arbitrary 8-dimensional spin vector bundle $\xi$ over $G_{4,2}(\mathbb{C})$ with the characteristic classes described in Example 4.3. An arbitrary element $R \in H^{4}\left(G_{4,2}(\mathbb{C}) ; \mathbb{Z}\right)$ has a form

$$
R=A c_{1}^{2}+B c_{2} .
$$

Condition (3) of Theorem 5.1 is satisfied, the other conditions (after minor simplification) read successively as

$$
\begin{gathered}
B \equiv 0 \bmod 2, \\
2 a A+b A+a B+b B-2 A^{2}-2 A B-B^{2} \equiv 0 \bmod 8, \\
b \equiv 0 \bmod 2, \\
C=2 a^{2}+2 a b+b^{2}+2 D, \\
2 a^{2}+2 a b+b^{2}-2 a-b-2 D+4 a A+2 b A+2 a B+2 B \equiv 0 \bmod 8 .
\end{gathered}
$$

The investigation of these conditions leads to the following result: An 8-dimensional spin vector bundle $\xi$ over $G_{4,2}(\mathbb{C})$ admits an $S p(2) \cdot S p(1)$-structure if and only if the following conditions are satisfied:

(1) $C=2 a^{2}+2 a b+b^{2}+2 D$,

(2) $2 \mid D$ and $4 \mid b$, 
(3) if $a \equiv 1 \bmod 4$ then $b+2 D \equiv 4 \bmod 8$,

(4) if $a \equiv 2 \bmod 4$ or $a \equiv 3 \bmod 4$ then $b+2 D \equiv 0 \bmod 8$.

EXAMPLE 5.5. The quaternionic projective space $\mathbb{H} P^{2}$ is also a quaternion-Kähler manifold. We will show that all the assumptions of Corollary 5.3 are satisfied. It can be seen from the following characteristic classes computed in $[\mathrm{BH}]$ :

$$
p_{1}\left(\mathbb{H} P^{2}\right)=2 u, \quad p_{2}\left(\mathbb{H} P^{2}\right)=7 u^{2}, \quad e\left(\mathbb{H} P^{2}\right)=3 u^{2}
$$

where $u \in H^{4}\left(\mathbb{H} P^{2} ; \mathbb{Z}\right)$ and $H^{*}\left(\mathbb{H} P^{2} ; \mathbb{Z}\right)=\mathbb{Z}[u] /\left\langle u^{3}\right\rangle$. On the other hand, it does not admit an $S p(2)$-structure, since (iii) of Corollary 4.7 is not satisfied. (It has not even almost complex structure, see [Hi].)

EXAMPLE 5.6. $G_{2} / S O(4)$ is the third 8-dimensional homogeneous space which is a quaternion-Kähler manifold. So it has an almost quaternionic structure. But in $[\mathrm{BH}]$ it is proved that $w_{6}\left(G_{2} / S O(4)\right) \neq 0$, which shows that the condition (b) in Corollary 5.2 is not necessary. According to Corollary 4.7, the same condition causes that $G_{2} / S O(4)$ does not admit an $S p(2)$-structure.

EXAMPLE 5.7. Complex 4-dimensional projective surfaces

$$
V_{d}=\left\{\left(z_{0}, z_{1}, \ldots, z_{5}\right) \in \mathbb{C} P^{5} ; z_{0}^{d}+z_{1}^{d}+\ldots+z_{5}^{d}=0\right\}
$$

considered as closed oriented smooth manifolds of real dimension 8 do not carry an $S p(2)$-structure. The equation

$$
\left\{4 p_{2}\left(V_{d}\right)-p_{1}^{2}\left(V_{d}\right)-8 e\left(V_{d}\right)\right\}\left[V_{d}\right]=d(d-2)(d-6)\left(-5 d^{2}+8 d-8\right)=0
$$

(condition (ii) in Corollary 4.7) has in positive integers the only solutions $d=2$ and $d=6$. But for both these $d$

$$
e\left(V_{d}\right)\left[V_{d}\right] \equiv 2 \bmod 4 .
$$

As for almost quaternionic structures, $V_{d}$ satisfies the necessary condition $(\mathrm{C})$ of Corollary 5.3 given by the equation above only if $d=2,6$. Since $V_{2}=G_{4,2}(\mathbb{C})$, we will deal only with $d=6$. We get

$$
p_{1}\left(V_{6}\right)=-30 c^{2}, p_{2}\left(V_{6}\right)=1095 c^{4}, e\left(V_{6}\right)=435 c^{4}, c_{1}\left(V_{6}\right)=0, c_{3}\left(V_{6}\right)=-70 c^{3},
$$

where $c \in H^{2}\left(V_{6} ; \mathbb{Z}\right)$ and $c^{4}\left[V_{6}\right]=6$. Hence all the assumptions of Corollary 5.3 are satisfied and $V_{6}$ has an almost quaternionic structure.

\section{References}

[AD] M. Atiyah and J. Dupont, Vector fields with finite singularities, Acta Math. 128 (1972), 1-40.

[BH] A. Borel and F. Hirzebruch, Characteristic classes and homogeneous spaces I, Amer. J. Math. 80 (1958), 458-538.

[Br] C. Brada, Eléments de la géométrie des octaves de Cayley, Thesis, Publications du Département de Mathématiques de l'Université de Lyon I, 2/d-1986.

[CV1] M. Čadek and J. Vanžura, On the classification of oriented vector bundles over 9complexes, Proceedings of the Winter School Geometry and Physics 1993, Suppl. Rend. Circ. Math. Palermo, Serie II 37 (1994). 
[CV2] M. Čadek and J. Vanžura, On 2-distributions in 8-dimensional vector bundles over 8-complexes, Colloq. Math. 70 (1996), 25-40.

[CV3] M. Čadek and J. Vanžura, On $S p(2)$ and $S p(2) \cdot S p(1)$-structures in 8-dimensional vector bundles, to appear in Publ. Math.

[CV4] M. Čadek and J. Vanžura, Almost quaternionic structures on eight-manifolds, to appear in Osaka J. Math. 35 (1) (1998).

[CV5] M. Čadek and J. Vanžura, On complex structures in 8-dimensional vector bundles, to appear in Manuscripta Math.

[CV6] M. Čadek and J. Vanžura, On 4-fields and 4-distributions in 8-dimensional vector bundles over 8-complexes, Colloq. Math. 76 (1998), 213-228.

[CS] M. C. Crabb and B. Steer, Vector-bundle monomorphisms with finite singularities, Proc. London Math. Soc. (3) 30 (1975), 1-39.

[Du] J. L. Dupont, K-theory obstructions to the existence of vector fields, Acta Math. 113 (1974), 67-80.

[E] C. Ehresmann, Sur les variétés presque complexes, Proceedings of the International Congress of Mathematicians II(1950), 412-419.

[Fr] H. Freudenthal, Octaven Ausnahmegruppen und Oktavengeometrie, Utrecht, 1951.

[GG] A. Gray and P. Green, Sphere transitive structures and the triality automorphism, Pacific J. Math. 34 (1970), 83-96.

[He] T. Heaps, Almost complex structures on eight- and ten-dimensional manifolds, Topology 9 (1970), 111-119.

[Hi] F. Hirzebruch, Komplexe Mannigfaltigkeiten, Proc. International Congress of Mathematicians 1958, Cambridge University Press, 1960, 119-136.

[HH] F. Hirzebruch and H. Hopf, Felder von Flächenelementen in 4-dimensionalen Mannigfaltigkeiten, Math. Ann. 136 (1958), 156-172.

[K1] U. Koschorke, Vector fields and other vector bundle morphisms - a singularity approach, Lecture Notes in Mathematics 847, Springer-Verlag, 1981.

[K2] U. Koschorke, Nonstable and stable monomorphisms of vector bundles, preprint (1995).

[Po] M. M. Postnikov, Lie Groups and Lie Algebras, Lectures in Geometry, Semester V, translation from Russian, Mir, Moscow, 1986.

[Q] D. Quillen, The mod 2 cohomology rings of extra-special 2-groups and the spinor groups, Math. Ann. 194 (1971), 197-212.

[T1] E. Thomas, Complex structures on real vector bundles, Am. J. Math. 89 (1966), 887-908.

[T2] E. Thomas, Vector fields on manifolds, Bulletin Amer. Math. Soc. 75 (1967), 643683.

[T3] E. Thomas, On the cohomology groups of the classifying space for the stable spinor group, Bol. Soc. Math. Mex. (2) 7 (1962), 57-69. 